\title{
Ibiza al descubierto: cambios en el consumo de drogas y en el comportamiento sexual de los jóvenes que pasan sus vacaciones en lugares conocidos internacionalmente por su vida nocturna*
}

\author{
Bellis, M.A.'; Hale, G. ${ }^{2}$; Bennet, A. ${ }^{3}$; Chaudry, M..3; Kilfoyle, M.' \\ (1) Public Health Sector. School of Health. John Moores University. Liverpool. \\ (2) Health Care Development (NHS North West) Manchester. \\ (3) HIT, Liverpool. \\ Enviar correspondencia: \\ Mark A. Bellis. School of Health. John Moores University. 70 Great Crosshall Street. Liverpool L.2AB. UK. m.a.bellis@livjm.ac.uk.
}

RESUMEN

Antecedentes: Crece el número de jóvenes que viajan a lugares de vacaciones donde se desconocen los niveles de consumo de drogas, sexo y otros riesgos asociados para la salud. Este estudio evalúa los cambios en los niveles del consumo de drogas y en el comportamiento sexual de jóvenes del Reino Unido después de una estancia en la isla de lbiza.

Métodos: Los datos se han obtenido a través de una encuesta realizada a una muestra transversal de jóvenes. Se entregaron los cuestionarios a 846 individuos, con edades entre los 15 y los 35 años, en el aeropuerto de Ibiza antes de su retorno al Reino Unido.

Resultados: Los jóvenes que visitan Ibiza modifican de forma significativa las pautas de consumo de drogas, alcohol y tabaco. En lo que se refiere al éxtasis, en el Reino Unido lo consumían cinco o más días a la semana el 2,9\% de los encuestados (9/313), mientras que en Ibiza llegaban a ser el 42,6\% (127/298). Menos individuos consumen otras drogas en Ibiza, aunque los que lo hacen consumen en mayores proporciones que cuando están en el Reino Unido ( $p<0.001)$. La mayoría de los jóvenes $(53,8 \%)$ tuvieron relaciones sexuales durante su estancia en Ibiza; el 26,2 \% sin utilizar preservativo y el $23,2 \%$ con más de una pareja. En líneas generales, el 7,3\% acudió a un hospital o a un médico durante su estancia en Ibiza, siendo los varones $(p<0.001)$, de más edad $(p<0.05)$ y en estancias más cortas $(p<0.005)$, los factores claves de riesgo.

Conclusiones: Es imprescindible la puesta en marcha de campañas urgentes y coordinadas de información para proteger la salud de los jóvenes, y se requiere para ello la cooperación europea a través de sus organizaciones, tanto públicas como privadas.

\section{ABSTRACT}

Background: Each year more people travel to international resorts where levels of substance use, sex and associated health risks are unknown. This study measures changes in levels of substance use and sexual behaviour of young people from the UK. when on holidays in Ibiza.

Methods: Data where collected through a cross-sectional survey collecting matched information on individuals' behaviour in the U.K. and abroad. Questionnaires were administered to 846 individuals aged between 15 and 35 at Ibiza Airport prior to their returning to the UK.

Results: Young visitors to Ibiza significantly alter patterns of drug, alcohol and tobacco use. For ecstasy, in the UK, 2,9\% of users (9/313) used five or more days a week while in Ibiza this rises to $42,6 \%$ (127/298). Fewer individuals use other drugs in Ibiza although those continuing to use in Ibiza consume at substantially higher rates than when in the UK $(p<0,001)$. Most individuals $(53,8 \%)$ had sex while in Ibiza; $26,2 \%$ had sex without a condom and $23,2 \%$ had more than one sexual partner. Overall, 7,3\% of individuals went to hospital or to see a doctor while in Ibiza with male $(p<0,001)$, older $(p<0,05)$ and staying for shorter times $(p<0,005)$ being the key risk factors.

Conclusions: Co-ordinated information campaigns to protect health of young people are urgently needed and will require European cooperation across both public and private organisations.

Keywords: drug use, sexual behaviour, travel, ecstasy, prevention, Ibiza.

Palabras clave: Consumo de drogas, conducta sexual, viajes, éxtasis, prevención, Ibiza.

\footnotetext{
*Artículo publicado previamente en Int. J. of Drug Policy 11, (2000) 235-244 con el título "Ibiza uncovered: changes in substance use and sexual behaviour amongst young people visiting an international night-life resort"
} 


\section{INTRODUCCIÓN}

E ncuestas recientes realizadas a jóvenes en el Reino Unido confirman el consumo excesivo de alcohol incluso entre los menores de 18 años (ONS, 1998; Hughes et al., 1997), describen la incapacidad de reducir los niveles de consumo de tabaco (Servicio Gubernamental de Estadística, 1998) y verifican un aumento en el consumo de drogas (Ramsey \& Partridge, 1999). Este espectacular aumento significa que, junto al elevado consumo de bebidas alcohólicas y tabaco, más de la mitad de los jóvenes entre 16 y 24 años también han probado alguna droga ilegal (aunque los consumidores habituales representan una menor proporción; Ramsey \& Partridge, 1999; Aldridge et al., 1999). Entre otros factores, el consumo regular de sustancias como el éxtasis, la cocaína y las anfetaminas ha sido asociado con la asistencia a lugares de ocio nocturnos y, recientemente, de forma más específica, a 'night-clubs' (Calafat et al., 1999; Kilfoyle \& Bellis, 1998; Forsyth et el., 1997).

El crecimiento reciente del fenómeno de los clubs en el Reino Unido (Garratt, 1998) significa que cada semana una gran cantidad de individuos, muchos de los cuales consumen drogas recreativas (Bean et al., 1997), acuden a altas horas de la noche a clubs o discotecas, y cada verano una proporción de éstos busca lugares en el extranjero donde se le ofrezcan similares oportunidades para bailar y divertirse (Sellars, 1998). Actualmente, se sabe muy poco acerca del consumo de drogas o del comportamiento sexual de los jóvenes que forman esos grupos de viaje, aunque las drogas y el sexo fuera de su país puedan acarrear riesgos desproporcionados para su salud. Por ejemplo, las drogas que se consiguen en estos lugares pueden ser menos fiables, aumentándose el riesgo de consumir sustancias desconocidas (Sherlock et al., 1999); climas más cálidos, en los que el peligro de infecciones gastrointestinales incrementan el riesgo de deshidratación (especialmente si se unen al consumo de drogas, tales como el éxtasis, 3,4- Methylenedioxymetanfetamina; McCann et al., 1996; Baker et al., 1997) y un enorme deseo de divertirse (Smeaton et al., 1998) pueden llevar a consumir toda clase de sustancias en exceso (Josiam et al.,1998). Además, medidas de protección básicas, tales como los preservativos, pueden ser difíciles de conseguir (Carter, 1997) para prevenir embarazos no deseados o niveles más altos de HIV en el caso de que se presente una inesperada oportunidad de practicar el sexo (Thomas et al., 1997). A diferencia de los grandes clubs del Reino Unido, algunos de estos lugares en el extranjero no disponen siquiera de normas de seguridad (Newcombe, 1994; London Drug Policy Forum, 1996), con el agua a precios prohibitivos en la barra de los clubs, escasa literatura para prevenir y atenuar los daños, y ausencia de un equipo de primeros auxilios, algo raramente disponible. Finalmente, cuando se presenta alguna emergencia, los sistemas sanitarios y judiciales se ven a menudo colapsados, por tratarse de lugares en los que una pequeña población nativa trata de acomodar a millones de personas.

Paradójicamente, para muchos jóvenes, el hecho de asumir esos riesgos supone escapar como individuos de las restricciones sociales que plantean la familia y el lugar de trabajo (Carter, 1997; Carter et al., 1997; Ryan \& Kinder, 1996). Para algunos grupos en vacaciones, el aumento del consumo de alcohol (Josiam et al.,1998) y un comportamiento sexual sin la debida protección (Thomas et al.,1997; Carter et al.,1997; Gillies et al.,1992) ha sido ya documentado. Sin embargo, ningún estudio ha descrito hasta la fecha el consumo de sustancias y el comportamiento sexual de los jóvenes cuando acuden a centros de vacaciones de animada vida nocturna en el extranjero. El presente estudio, así, tiene como objetivo evaluar los cambios en estos comportamientos de riesgo cuando los jóvenes del Reino Unido pasan sus vacaciones anuales en rincones de esas características en cualquier lugar de Europa.

Entre estos lugares elegidos por los jóvenes, Ibiza es indiscutiblemente el más popular de Europa por sus 'night-clubs'. Esta isla balear se beneficia de una larga asociación entre la música de discoteca y el consumo de droga recreativa, especialmente éxtasis (Calafat et al., 1998). En 1986, esa sustancia fue lanzada desde Ibiza como la droga idónea para las discotecas, descrita como el perfecto acompañamiento a un tipo de música llamado "beat balear" (Calafat et al., 1998). La popularidad de Ibiza ha crecido desde entonces, ayudada por los vuelos internacionales baratos y los paquetes vacacionales dirigidos fundamentalmente al mercado juvenil. Como consecuencia de ello, Ibiza atrajo entre enero y septiembre de 1999 alrededor de 700.000 turistas sólo del Reino Unido, y una isla, con una población endémica de 80.000 personas, recibió a más de un millón y medio de turistas (Fomento del Turismo de Ibiza, 1999). Por ese motivo, decidimos elegir esta isla como el marco más adecuado para realizar nuestro estudio.

\section{MÉTODOS}

Se preparó un breve cuestionario anónimo para evaluar las variables demográficas básicas, el consumo de alcohol, tabaco y otros siete tipos de droga durante su estancia en Ibiza (anfetamina, ketamina, cannabis, éxtasis, LSD, cocaína y GHB), y las pautas de consumo durante los seis meses previos en el Reino Unido. Se preguntaba, además, el comportamiento sexual en Ibiza y en su país de origen, los contactos con los servicios sanitarios durante su estancia en la isla y la duración y motivación de su visita. Final- 
mente, se indagaba si durante su estancia en la isla se les había entregado alguna información relacionada con el consumo de drogas.

Para captar los detalles del comportamiento de los individuos durante su permanencia en la isla, se entregaron cuestionarios en las zonas de salida del aeropuerto de lbiza a jóvenes (con una edad máxima de 35 años) que se hallaban en las colas de facturación antes de embarcar hacia el Reino Unido. Se les preguntaba previamente si disponían de tiempo para contestar a un pequeño cuestionario ( $n=1076)$. A aquellos que contestaban afirmativamente $(n=901$, el $83,7 \%$ de los abordados) se les explicaban el tema y los detalles del estudio, pasándoles a continuación las preguntas. Aunque 870 cuestionarios (el 96,6\% de los distribuidos) se devolvieron cumplimentados, sólo 846 (el 93,8\% de los distribuidos) fueron válidos, además de contestados por individuos dentro de la franja de edad pertinente. La distribución de los cuestionarios comenzó el 8 de julio de 1999 y terminó el 2 de septiembre de ese mismo año. Todos los cuestionarios se enviaron a Inglaterra para entrar en una base de datos que permitiría su posterior estudio con SPSS (SPSS, 1996). En todos los análisis se utilizaron estadísticas no paramétricas cuando los datos no habían sido distribuidos normalmente y análisis de regresión logística siempre que las correlaciones entre variables hubieran podido de otro modo enmascarar los resultados.

\section{RESULTADOS}

En total, 456 varones y 390 mujeres completaron los cuestionarios. La mayor parte de estos individuos habían residido durante una semana $(50,4 \%)$ o dos $(44,5 \%)$ en San Antonio (92,3\%), el centro de vacaciones más importante de la isla. Las edades iban desde los 15 hasta los 35 años, con una edad media de 20 años (varones, 20 años; mujeres, 19 años). El principal motivo para elegir Ibiza incluía en primer lugar la música de las discotecas (78\%) y el clima (el 49,6\%), y a menos individuos les había atraído el sexo $(23,6 \%)$, las drogas $(17,2 \%)$ o el trabajo $(1,7 \%)$. Los chicos habían elegido el sexo como motivo de la visita más a menudo que las chicas (varones, 33,6\%; hembras, $11,9 \% ; p 2=54,2, p=0,001)$. No había otros factores de la selección significativamente diferentes entre hombres y mujeres. Para el 53,0\% de individuos se trataba de su primera visita a Ibiza. Los que repetían el viaje a la isla identificaban marginalmente el consumo de drogas como razón más probable de su visita a Ibiza (primera visita, 14,3\%; visitas posteriores, 20,3\%; A2 (tendencia) $=5.211, p<0.05$ ) .

\section{Consumo de drogas.}

Los porcentajes de individuos que consumen diferentes drogas en Ibiza y en los seis meses precedentes (en el Reino Unido) se muestran en el cuadro 1 junto a las comparaciones estadísticas entre la frecuencia del consumo de una sustancia en cada uno de los dos lugares. Los individuos mostraban tendencia a consumir menos drogas durante su estancia en Ibiza que en los seis meses previos en Inglaterra, si exceptuamos el alcohol, el tabaco y el éxtasis, donde las proporciones del consumo por parte de los individuos en Ibiza no difería significativamente del de los últimos seis meses en el Reino Unido. Mientras el número de individuos que consumía la mayoría de las drogas disminuía (Tabla 1), cuando lo hacían, la frecuencia del consumo de todas las sustancias era significativamente más alta en Ibiza que en el Reino Unido (Tabla 2). Así, mientras solamente el 2,9\% de los usuarios de éxtasis lo consumían en su país cinco o más veces por semana, el 42,6\% de los consumidores de éxtasis lo hacían en lbiza cinco o más veces por semana. Se contemplaron incrementos similares

Tabla 1. Sustancias consumidas mientras se estaba de vacaciones en lbiza y durante los seis meses anteriores en el Reino Unido

\begin{tabular}{|lcccccc|}
\hline Sustancia & $n$ & \multicolumn{2}{l}{ Porcentaje que usa drogas } & McNemar $P$ \\
\cline { 3 - 6 } & & No uso & Sólo en UK & Sólo en lbiza & Ambos sitios & \\
\hline Alcohol & 842 & 1.2 & 0.7 & 0.6 & 97.5 & 1 \\
Tabaco & 842 & 36.8 & 3.4 & 4.6 & 55.1 & 0.275 \\
Anfetas & 842 & 68.4 & 17.7 & 2.3 & 11.6 & $<0.001$ \\
Ketamina & 842 & 90.5 & 4.5 & 1.8 & 3.2 & $<0.005$ \\
Cannabis & 842 & 49.8 & 15.2 & 1.9 & 33.1 & $<0.001$ \\
Extasis & 842 & 59.5 & 5.2 & 3.3 & 31.9 & 0.076 \\
LSD & 841 & 87.5 & 9.0 & 0.7 & 2.7 & $<0.001$ \\
Cocaina & 842 & 77.0 & 8.1 & 2.0 & 12.9 & $<0.001$ \\
GHB & 842 & 96.0 & 2.5 & 0.7 & 0.8 & $<0.01$ \\
\hline
\end{tabular}




\begin{tabular}{|c|c|c|c|c|c|c|}
\hline Sustancia & Lugar & $\begin{array}{l}<1 \text { día } \\
\text { semana (\%) }\end{array}$ & $\begin{array}{l}1 \text { día por semana } \\
(\%)\end{array}$ & $\begin{array}{l}\text { 2-4 días semana } \\
(\%)\end{array}$ & $\begin{array}{l}>5 \text { días semana } \\
(\%)\end{array}$ & $\begin{array}{l}\mathrm{X}^{2} \text { (tend.) } P \\
\text { value }\end{array}$ \\
\hline Alcohol & $\begin{array}{l}\text { UK } \\
\text { Ibiza }\end{array}$ & $\begin{array}{l}6.5 \\
1.1\end{array}$ & $\begin{array}{r}16.2 \\
2.4\end{array}$ & $\begin{array}{r}52.5 \\
9.1\end{array}$ & $\begin{array}{r}24.8 \\
87.4\end{array}$ & $\begin{array}{l}482.5 \\
<0.001\end{array}$ \\
\hline Tabaco & $\begin{array}{l}\text { UK } \\
\text { Ibiza }\end{array}$ & $\begin{array}{r}12.8 \\
3.6\end{array}$ & $\begin{array}{l}5.3 \\
5.3\end{array}$ & $\begin{array}{l}8.7 \\
7.5\end{array}$ & $\begin{array}{l}73.2 \\
83.6\end{array}$ & $\begin{array}{l}24.48 \\
<0.001\end{array}$ \\
\hline Anfetamina & $\begin{array}{l}\text { UK } \\
\text { Ibiza }\end{array}$ & $\begin{array}{l}79.8 \\
18.8\end{array}$ & $\begin{array}{l}13.8 \\
23.9\end{array}$ & $\begin{array}{r}4.5 \\
32.5\end{array}$ & $\begin{array}{r}2.0 \\
24.8\end{array}$ & $\begin{array}{l}138.9 \\
<0.001\end{array}$ \\
\hline Ketamina & $\begin{array}{l}\text { UK } \\
\text { Ibiza }\end{array}$ & $\begin{array}{l}81.5 \\
14.3\end{array}$ & $\begin{array}{l}12.3 \\
33.3\end{array}$ & $\begin{array}{r}1.5 \\
26.2\end{array}$ & $\begin{array}{r}4.6 \\
26.2\end{array}$ & $\begin{array}{l}39.9 \\
<0.001\end{array}$ \\
\hline Cannabis & $\begin{array}{l}\text { UK } \\
\text { Ibiza }\end{array}$ & $\begin{array}{r}40.3 \\
8.8\end{array}$ & $\begin{array}{r}8.6 \\
11.5\end{array}$ & $\begin{array}{l}21.9 \\
25.8\end{array}$ & $\begin{array}{l}29.2 \\
53.9\end{array}$ & $\begin{array}{l}80.7 \\
<0.001\end{array}$ \\
\hline Extasis & $\begin{array}{l}\text { UK } \\
\text { Ibiza }\end{array}$ & $\begin{array}{l}60.7 \\
10.4\end{array}$ & $\begin{array}{r}25.2 \\
11.7\end{array}$ & $\begin{array}{r}11.2 \\
35.2\end{array}$ & $\begin{array}{r}2.9 \\
42.6\end{array}$ & $\begin{array}{l}260.7 \\
<0.001\end{array}$ \\
\hline LSD & $\begin{array}{l}\text { UK } \\
\text { Ibiza }\end{array}$ & $\begin{array}{r}90.9 \\
27.6\end{array}$ & $\begin{array}{r}5.1 \\
10.3\end{array}$ & $\begin{array}{r}3.0 \\
31.0\end{array}$ & $\begin{array}{r}1.0 \\
31.0\end{array}$ & $\begin{array}{l}56.3 \\
<0.001\end{array}$ \\
\hline Cocaina & $\begin{array}{l}\text { UK } \\
\text { Ibiza }\end{array}$ & $\begin{array}{l}78.0 \\
14.2\end{array}$ & $\begin{array}{r}9.6 \\
30.7\end{array}$ & $\begin{array}{r}5.6 \\
33.9\end{array}$ & $\begin{array}{r}6.8 \\
21.3\end{array}$ & $\begin{array}{l}90.3 \\
<0.001\end{array}$ \\
\hline GHB & $\begin{array}{l}\text { UK } \\
\text { lbiza }\end{array}$ & $\begin{array}{r}78.6 \\
7.7\end{array}$ & $\begin{array}{r}3.6 \\
30.8\end{array}$ & $\begin{array}{l}10.7 \\
23.1\end{array}$ & $\begin{array}{r}7.1 \\
38.5\end{array}$ & $\begin{array}{l}13.3 \\
<0.001\end{array}$ \\
\hline
\end{tabular}

en la frecuencia del consumo de todas las drogas. Para investigar si todo ello era resultado de un cambio en el comportamiento de los individuos (más que un subset diferente del consumidor más duro al hallarse en Ibiza) con respecto a cada droga, los análisis se centraron en aquellos que las consumían en los dos lugares (Tabla 3). En cuanto al aumento de frecuencia en el consumo en Ibiza, resulta muy significativo, por ejemplo, que los que consumían anfetaminas cinco o más veces por semana, pasaba de ser un $4,1 \%$ en el Reino Unido a un $26,5 \%$ en Ibiza; en cuanto al éxtasis, al aumento equivalente subía de un $3,0 \%$ a un $45,0 \%$

\begin{tabular}{|c|c|c|c|c|c|c|c|c|}
\hline \multirow[t]{2}{*}{ Sustancia } & \multirow[t]{2}{*}{$N$} & \multicolumn{2}{|c|}{ Frecuencia de uso media } & \multicolumn{2}{|c|}{$\begin{array}{l}\% \text { usando } 5 \text { o más veces } \\
\text { por semana }\end{array}$} & \multirow{2}{*}{$\begin{array}{l}\% \text { Aum. } \\
\text { su uso } \\
\text { en Ibiza }\end{array}$} & \multirow{2}{*}{$\begin{array}{l}\% \text { Dism. } \\
\text { su uso } \\
\text { en Ibiza }\end{array}$} & \multirow{2}{*}{$\begin{array}{l}\text { Wilcoxom } \\
P^{*}\end{array}$} \\
\hline & & $\begin{array}{c}\text { Días por sem. } \\
\text { UK }\end{array}$ & $\begin{array}{l}\text { Días por sem. } \\
\text { Ibiza }\end{array}$ & UK & Ibiza & & & \\
\hline Alcohol & 821 & $2-4$ & $\geq 5$ & 24.7 & 87.3 & 68.2 & 1.7 & $<0.001$ \\
\hline Tabaco & 464 & $\geq 5$ & $\geq 5$ & 76.3 & 87.3 & 19.2 & 1.7 & $<0.001$ \\
\hline Anfetas & 98 & 1 & $2-4$ & 4.1 & 26.5 & 29.6 & 0.0 & $<0.001$ \\
\hline Ketamina & 27 & 1 & $2-4$ & 7.4 & 29.6 & 74.1 & 0.0 & $<0.001$ \\
\hline Cannabis & 279 & $2-4$ & $\geq 5$ & 39.4 & 55.6 & 42.3 & 11.5 & $<0.001$ \\
\hline Extasis & 269 & $<1$ & $2-4$ & 3.0 & 45.0 & 84.4 & 1.9 & $<0.001$ \\
\hline LSD & 23 & $<1$ & $2-4$ & 0.0 & 30.4 & 69.6 & 0.0 & $<0.001$ \\
\hline Cocaina & 109 & $<1$ & $2-4$ & 7.3 & 22.0 & 71.6 & 4.6 & $<0.001$ \\
\hline GHB & 7 & 1 & $2-4$ & 14.3 & 14.3 & 71.4 & 0.0 & $<0.05$ \\
\hline
\end{tabular}


(Tabla 3). El número de pastillas de éxtasis tomadas en una noche habitual no difería significativamente entre las dos localidades (Ibiza, mediana $=2$; Reino Unido, mediana $=2 ; Z=0,421, p=0,673)$. A pesar de esos niveles de consumo, sólo una tercera parte $(37,5 \%)$ de los individuos tuvo acceso a alguna información sobre drogas relacionada con la salud.

\section{Comportamiento sexual}

La mayor parte (el 84\%) de los visitantes de Ibiza declararon haber tenido por lo menos una pareja sexual en los seis meses anteriores (Reino Unido), mientras un 53,8\% afirmaron haber tenido por lo menos una pareja sexual durante su estancia en Ibiza. No había ninguna diferencia significativa entre sexos en el número que declaraba haber tenido por lo menos una pareja en el Reino Unido (P2=0,02, $P=0,883)$. Sin embargo, los hombres eran menos reticentes que las mujeres a declarar haber tenido al menos una pareja en lbiza (varones, 57,6\%, mujeres, 49,5\%, P2=5.418, p<0,05). Utilizando un análisis de regresión logística, los efectos de género desaparecen y, en su lugar, el número de parejas tenidas en el Reino Unido (últimos seis meses) y la duración de la estancia en Ibiza son los dos únicos factores relevantes positivamente asociados con la práctica del sexo en Ibiza (parejas en el Reino Unido $p<0,001$, días en Ibiza $p<0,005)$. Las pautas de consumo de drogas no estaban asociadas significativamente con el hecho de tener relaciones sexuales en Ibiza. El cuadro 4 muestra el número de parejas sexuales de los encuestados tanto en el Reino Unido como en Ibiza. El número de parejas habidas en los últimos seis meses en el Reino
Unido tenía una correlación significativa con dicho número en Ibiza ( $r$-spearman $=0,336, p<0,001$ ). Una vez más, a pesar de que los diferentes sexos declaraban un número diferente de parejas en Ibiza $(Z=3,78$, $p<0,001)$, utilizando la regresión logística el efecto desaparecía al ser controlada la actividad sexual en el Reino Unido. Más de la cuarta parte de todos los que fueros consultados (el 26,2\%, ver tabla 4b) tuvieron relaciones sexuales sin usar preservativo por lo menos una vez durante su estancia en Ibiza. Entre aquellos usuarios de sexo en Ibiza, la falta de protección iba asociada positivamente con el hecho de ser varón $(p<0,05)$ y el número de parejas sexuales habidas en Ibiza ( $p<0,05$, análisis de regresión logística, ver tabla 4b). De nuevo, ninguna de las pautas de consumo de drogas en lbiza aparecía relacionada significativamente con la actividad sexual sin preservativos.

Finalmente, un 7,3\% de individuos tuvieron que ir al hospital o a visitar a un médico durante su estancia en Ibiza. No se preguntó en este estudio la razón de tales visitas. Sin embargo, los análisis de regresión logística revelaron que los hombres $(p<0,001)$, mayores $(p<0,05)$ y con estancias más cortas $(p<0,005)$ habían tenido menos reparos en solicitar atención médica.

\section{DISCUSIÓN}

Los resultados de este estudio indican que las pautas de consumo de drogas de los jóvenes difieren sustancialmente de las habituales en el Reino Unido

Tabla 4. Compañeros sexuales en lbiza estratificados según el número de compañeros en UK.

\begin{tabular}{|c|c|c|c|c|c|}
\hline \multirow[t]{2}{*}{ Compañeros en Ibiza } & \multicolumn{4}{|c|}{ Compañeros en UK (últimos 6 meses) } & \multirow[t]{2}{*}{ Total } \\
\hline & 0 & 1 & $2-5$ & $\geq 6$ & \\
\hline \multicolumn{6}{|c|}{ a) Número de Individuos } \\
\hline 0 & 93 & 178 & 102 & 8 & 381 \\
\hline 1 & 23 & 153 & 71 & 5 & 252 \\
\hline $2-5$ & 11 & 35 & 73 & 17 & 136 \\
\hline$\geq 6$ & 4 & 11 & 13 & 27 & 55 \\
\hline Total & 131 & 377 & 259 & 57 & 824 \\
\hline \multicolumn{6}{|c|}{$\begin{array}{l}\text { b) Porcentaje de cada categoría que han tenidos relaciones sexuales } \\
\text { en Ibiza al menos una vez sin utilizar preservativo. }\end{array}$} \\
\hline 0 & 0.0 & 0.0 & 0.0 & 0.0 & 0.0 \\
\hline 1 & 26.1 & 62.1 & 42.3 & 40.0 & 52.8 \\
\hline $2-5$ & 9.1 & 37.1 & 39.7 & 23.5 & 34.6 \\
\hline$\geq 6$ & 75.0 & 54.5 & 61.5 & 70.4 & 65.5 \\
\hline Total & 7.6 & 30.2 & 25.9 & 43.9 & 26.2 \\
\hline
\end{tabular}


cuando pasan sus vacaciones en el extranjero (tablas 1-3).

Actualmente, menos individuos toman anfetaminas, ketaminas, cannabis, LSD, cocaína y GHB en unas vacaciones típicas en Ibiza que en los últimos seis meses en casa. Esto podría representar la elección de evitar ciertas sustancias en el extranjero. Sin embargo, el mercado en Ibiza puede estar relativamente poco desarrollado (Hopkins, 1999) y el consumo de menos drogas comunes puede deberse a una oferta limitada. El consumo no parece menor cuando se trata de drogas más conocidas, como alcohol, tabaco y éxtasis (tabla 1). Además, cuando los individuos se las arreglan para obtener drogas (presumiblemente llevándolas clandestinamente a la isla o consiguiéndolas en la propia Ibiza), la frecuencia en el consumo aumenta de forma espectacular. Así, el consumo de éxtasis en Ibiza se caracteriza por aumentar quince veces la proporción de usuarios que la consumen cinco o más veces por semana. En realidad, la frecuencia en el consumo se incrementó en todas las drogas; por ejemplo, quienes tomaron anfetaminas declararon tener doce veces más tendencia a consumir cinco o más veces por semana dicha droga en Ibiza que en el Reino Unido (tabla 2). Aunque las comparaciones de cada droga se limiten a los consumidores de ésta en los dos países (tabla 3), la frecuencia media de consumo aumenta en todas las drogas; en cuanto al éxtasis, éste también pasa de ser utilizado menos de una vez por semana en el Reino Unido a dos y hasta cuatro veces por semana en Ibiza (tabla 3).

Las cantidades de éxtasis en cada toma eran semejantes en ambos lugares, con una media de dos pastillas al día. Sin embargo, mientras en el Reino Unido esto representaría sólo el 2,9\% de usuarios (tabla 2), ingiriendo 10 o más pastillas a la semana, en Ibiza más del $40 \%$ de los que continuaban con éxtasis tomaba 10 o más pastillas a la semana, a veces durante un período de dos semanas. Por otra parte, un $31,9 \%$ de los visitantes de la isla consumieron más de una droga (excluyendo el alcohol y el tabaco) durante su estancia. De los que tomaron éxtasis, por ejemplo, casi un tercio (el 32,2\%) consumió también anfetaminas y prácticamente todos bebieron alcohol (el $97,3 \%)$. Los efectos toxicológicos potenciales del abuso de la mezcla de diferentes drogas tomadas con esa frecuencia, incluso en períodos cortos de una o dos semanas, están aún por estudiar. Además, el éxtasis puede, por ejemplo, contener realmente otras sustancias (Sherlock et al., 1999). Esto representa un riesgo adicional, pues se experimentan cócteles diferentes de compuestos desconocidos con efectos sorprendentes. A pesar de esos peligros, sólo una tercera parte de los encuestados recibió alguna información sobre drogas relacionada con la salud durante su estancia en la isla, y, por consiguiente, muchos ignoraban los riesgos potenciales para la salud o qué debían hacer en caso de presentarse alguna emergencia.

Mientras este estudio, a diferencia de otros (Josiam et al., 1998; Graves, 1995), no encontró relación entre las pautas de consumo de drogas y el comportamiento sexual, incluso las cifras de individuos que practican el sexo sin protección en Ibiza resultaron preocupantes. Aunque solamente un $23,6 \%$ de los jóvenes indicó haber acudido a la isla en busca de sexo, más de la mitad tuvieron relaciones sexuales durante su estancia en ella, y el 48,4\% de éstos lo hicieron sin preservativo al menos con una persona (Tabla 4a,b); a pesar de que para algunos individuos ésta pudo haber sido su pareja estable -que pasaba las vacaciones con él-, para muchos, incluyendo a los que tuvieron varias parejas en Ibiza (23,2\% de los encuestados, tabla 4a), éstas representaron nuevos contactos sexuales.

A menudo, los paquetes vacacionales de lugares como Ibiza ofrecen largas noches llenas de alcohol y la posibilidad de nuevas parejas sexuales. Por ejemplo, el Club 18-30, uno de los más famosos tour operadores para jóvenes, incluye en su publicidad "Prueba el sexo en la cresta de la ola... Bebe todo tu 'duty free' en un día" (Club 18-30, 1999). En el presente estudio se han confirmado elementos significativos de actividad sexual y un consumo de alcohol muy elevado, pero asociados al aumento del consumo de drogas recreativas. La capacidad de esa clase de lugares para controlar el tráfico de drogas es limitada y, con el elevado número de usuarios recreacionales que visitan actualmente Ibiza sin consumir drogas (tabla 1), queda un considerable potencial para el tráfico de sustancias y el crecimiento de su consumo en el futuro. A corto plazo, medidas para minimizar el daño como folletos (Health Education Authority, 1999; HIT \& LJMU, 1999), información para poder buscar la adecuada asistencia médica en el extranjero y un fácil acceso a los preservativos parecen pasos esenciales para proteger la salud de los jóvenes durante sus vacaciones. Este material educativo y un buen asesoramiento deberían reflejar ciertos cambios, como hemos señalado aquí, en el consumo de drogas asociado a lo que se ha denominado: "experiencia turística con baile y música" (Sellars, 1998). Es necesario que los materiales y los mensajes destinados a ser utilizados en el extranjero incluyan algunos consejos sobre el consumo de las drogas más frecuentes, los riesgos de consumirlas mezcladas y adulteradas, los peligros de deshidratación, el acceso a medidas de protección de la salud, tales como preservativos, y las dificultades para acceder a servicios médicos. Al menos en parte, la responsabilidad de la distribución de ese material debería recaer en las compañías de turismo, líneas aéreas, hoteles, clubs nocturnos y otros lugares que pueden ayudar a proteger la salud de sus clientes (Mc Kee, 1996). Por otra parte, los centros de reunión de 
los jóvenes y las grandes marcas deberían colaborar con estas medidas e impulsar el uso de medidas protectoras como los preservativos (Bellis\& Ashton, 2000).

Numerosos jóvenes han reservado ya para el año 2000 plazas de vacaciones en Ibiza y en otros lugares europeos de animada vida nocturna. Actualmente, el coste a largo plazo para la salud de dosis habituales, incluso pequeñas, de drogas como el éxtasis, son inciertos (Jansen, 1999; McCann et al., 1999; Green \& Goodwin, 1996), y los efectos a largo plazo de los encuentros fugaces e intensivos con la droga comentados en este informe (tablas 2 y 3) seguirán siendo pura especulación durante algún tiempo. Asimismo, aunque esta investigación señala cambios significativos en el consumo de drogas de los jóvenes durante sus vacaciones fuera del país nativo, es necesario realizar con urgencia más estudios de investigación para examinar los cambios que se efectúen a largo plazo en el comportamiento del consumo de sustancias, que puede persistir tras la vuelta de los individuos al Reino Unido. Mientras tanto, sin embargo, existe la posibilidad de asegurar que hay una serie de medidas preventivas disponibles para suministrar -a individuos que eligen proteger su salud en vacaciones de ese tipo- toda la información pertinente (preservativos, agua barata, etc..). Tales iniciativas ayudarán a minimizar los riesgos de incidentes relacionados con drogas, a reducir las posibles repercusiones para la salud, y a fomentar, al menos, el sexo seguro. Esas medidas, sin embargo, requerirán, no sólo cooperación entre los organismos de salud pública de los diferentes países europeos, sino también un más alto nivel de colaboración con los organismos comerciales que operan en el mundo del turismo especializado en música y diversión.

\section{AGRADECIMIENTOS}

El estudio no hubiera sido posible sin la ayuda de NHS (North West) y, en particular, del profesor John Ashton y del señor Rod Thomson. También queremos dar las gracias a Ben y a James por su trabajo de apoyo en lbiza, a los participantes en el estudio que sacrificaron tiempo de sus vacaciones para hacerlo posible y al doctor Bennett Lee por sus observaciones sobre el manuscrito.

\section{BIBLIOGRAFÍA}

Aldridge J, Parker H, Measham F. Drug Trying and Drug Use Across Adolescence: a longitudinal study of young people's drug taking in two regions on northern England. London: Drugs Prevention Advisory Service, Home Office 1999.

Baker O, Chistian G, Farrel M, Finch E, Ford J, Morgan G, Sell L. The Misuse of Drugs. Netherlands: British Medical Association, 1997.

Bellis MA \& Ashton JR. Commercial advertisements are needed to create a condom culture. BMJ. 2000; 320:643.

Bean P, Stratford N, White C, Goodman M, Malyon T, Charles V, O'Hagan C. Release. Drugs and Dance Survey; an insight into the culture. London: Release 1997.

Calafat A, Bohrn K, Juan M, Kokkevi A, Maalste N, Mendes F, Palmer A. Nightlife in Europe and recreative drug use. SONAR98. Palma: IREFREA \& European Commission. 1999.

Calafat A, Stocco P, Mendes F, Simon J, van de Wijngaart G, Sureda M, Palmer A, Maalste N, Zavatti P. Characteristics and social representation of ecstasy in Europe. Valencia: IREFREA \& European Commission, 1998.

Carter S. Taking sexual risks while away from home. MRC news. 1997; Autumn, 38-9.

Carter S, Horn K, Hart G, Dunbar M, Scoular A, Maclntyre $S$. The sexual behaviour of international travellers at two Glasgow GUM clinics. Inter. J. STD AIDS. 1997; 8: 336-8.

Club 18-30. Budget Edition, Summer 1999, Mission Statement. Club 18-30, 1999.

Department of Health. Health information for Overseas Travel. London: HMSO, 1995.

Fomento del Turismo de Ibiza, Ibiza 1999 tourist statistics, Palma, 1999.

Forsyth, AJM, Barnard M, McKeganey NP. Musical preference as an indicator of adolescent drug use. Addiction 1997; 10: 1317-25.

Garratt S. Adventures in Wonderland: A decade of club culture. London: Headline 1998.

Gillies P, Slack PA, Stoddart N, Conway S. HIV-related risk behaviour in UK holidaymakers. AIDS 1992; 6: 339-42.

Government Statistical Service. Statistics on Smoking: England, 1976 to 1996. London: Department of Health, 1998.

Graves KL. Risky sexual behaviour and alcohol use among young adults: results from a national survey. Am. J. Health Promot.1995; 10: 27-36.

Green A.R., Goodwin G.M.: Ecstasy and neurodegeneration; ecstasy's log term effects are potentially more damaging than its acute toxicity. 1996; BMJ. 312: 1493-4.

Health Education Authority. Drugs abroad; essential holiday information. London; HEA, 1999. 
HIT \& Liverpool John Moores University. Club Health Ibiza. Liverpool; Splinter, 1999.

Hopkins N. High anxiety. Guardian, 26 th August 1999.

Hughes K, MacKintosh AM, Hastings G, Wheeler C, Watson J, Inglis J. Young people, alcohol and designer drinks: quantitative and qualitative study. BMJ 1997; 314: 414-8.

Jansen KL Ecstacy (MDMA) dependence. Drug Alcohol Depend.1999; 53: 121-4.

Josiam BM, Perry Hobson JS, Dietrich UC Smeaton G. An analysis of the sexual, alcohol and drug related behavioural patterns of students on spring break. Tour. Manag. 1998; 19: 501-13.

Kilfoyle M, Bellis MA. The Health of the Clubbing Nation. In Kilfoyle M, Bellis MA eds. Club Health. Liverpool: Molyneux associates. 1998.

London Drug Policy Forum. Dance till dawn safely. London: London Drug Policy Forum, 1996.

McCann, U. D.; Mertl, M.; Eligulashvili, V.; Ricaurte, G. A.: Cognitive performance in $(+/-) 3,4-$ Methylenedioxymethamphetamine (MDMA; 'ecstasy') users: a controlled study. Psychopharma. 1999; 143: 417-25.

McCann UD, Slate SO, Ricaurte GA Adverse reactions with 3,4 - Methylenedioxymethamphetamine (MDMA; 'ecstasy'). Drug Safety 1996; 15: 107-

McKee M. Travel associated illness. BMJ 1996; 312: 925-6.
Newcombe R. Safer Dancing: guidelines for good practice at dance parties and night clubs. Manchester: Lifeline, 1994.

Office for National Statistics. Living in Britain: results from the 1996 General Household Survey. London: The Stationary Office, 1998.

Ramsey M, Partridge S. Drug Misuse Declared in 1998: results from the British Crime Survey. London: Home Office, 1999.

Ryan C, Kinder R. Sex, tourism and sex tourism: fulfilling similar needs? Tour. Manag. 1996; 17: 50718.

Sellars A. The influence of dance music on the UK youth tourism market. Tour. Manag. 1998; 19: 61115.

Sherlock K. Wolff K, Hay AW, Conner M. Analysis of illicit ecstasy tablets: implications for clinical management in the accident and emergency department. J. Accid. Emerg. Med. 1999; 16: 194-7.

Smeaton GL, Josiam BM Dietrich UC. College students' binge drinking at a beach-front destination during spring break. College Health 1998; 46: 247-54.

SPSS, SPSS Base 7 Advanced Statistics Guide, USA, SPSS, 1996.

Thomas M, Bloor M, Crosier A. Young people and international travel; HIV prevention and health promotion. London: Health Education Authority, 1997. 